\title{
Envenenamiento por Paraquat y hemoperfusión con carbón activado
}

\author{
A. M. LÓPEZ LAGO, C. RIVERO VELASCO, C. GALBAN RODRÍGUEZ, \\ A. MARIÑO ROZADOS, N. PIÑEIRO SANDE, E. FERRER VIZOSO \\ Servicio de Medicina Intensiva. Hospital Clínico Universitario de Santiago de \\ Compostela
}

\author{
PARAOUAT INTOXICATIONS AND HEMOPERFUSION WITH ACTI - \\ VATED CHARCOAL
}

\section{RESUMEN}

El Paraquat es un herbicida del grupo de los biperidilicos, de los más utilizados en nuestro pais.

En España existen muy pocos casos descritos de esta intoxicación y presenta una alta mortalidad incluso con la ingestión de mínimas cantidades.

Presentamos dos casos de intoxicación por ingesta accidental de este producto, que llegan a nuestro hospital en las tres horas posteriores. Se instaura tratamiento con lavado gástrico, carbón activado, $\mathrm{N}$ acetil cisteina, tierra de Fuller, catárticos, medidas de apoyo y hemoperfusión con carbón activado; consiguiendose niveles indetectables del tóxico 48 horas después y mejorando su sintomatología, predominantemente gastrointestinal.

Se comentan los casos clínicos durante su estancia en U.C.I así como aspectos clínicos de estas intoxicaciones, su tratamiento actual y últimas tendencias y factores pronósticos.

PALABRAS CLAVE: Intoxicación por Paraquat.

\begin{abstract}
The Paraquat is a herbicide more using in Spain. In our country the re are a few cases this intoxication and it presents a hight mortality even if the patients ingest a minimal amount.

We present two cases of casual poisoning with paraquat. These patients were admited three hours after ingestion of toxic. They were tre ated with with orogastric lavage, activated charcoal, $N$-acetil-cysteina, Fuller's earth, catartics, support measuring and hemperfusion with acti vated charcoal, with this treatments both patients had a indetectable levels of paraquat 48 hours after and yours symptoms that was gastric and intestinal predominantly had missing. We present the graphics of evolution the plasmatic and urine levels of paraquat the both patients.

We review the differents aspects of treatment and update of this into xication.
\end{abstract}

KEY WORDS: Paraquat intoxications.

López Lago AM, Rivero Velasco C, Galban Rodríguez C, Mariño Rozados A, Piñeiro Sande N, Ferrer Vizoso E. Envenenamiento por Paraquat y hemoperfusión con carbon activado. An Med Interna (Madrid) 2002; 19: 310-312.

\section{INTRODUCCIÓN}

El Paraquat es un herbicida biperidílico (1,1' dimetil-4,4' diperidilo) muy ampliamente utilizado en nuestro país y con pocos riesgos si se adopatan las precauciones adecuadas. $\mathrm{Su}$ intoxicación sin embargo es letal con muy pequeñas dosis del producto, llegando a mortalidad del $100 \%$ en algunas series (1).

La mayoría de las intoxicaciones se producen por ingestión oral, pero pueden producirse por vía dérmica, aunque la absorición por esta vía es mínima a menos que se trate de una exposición muy prolongada con altas concentraciones; puede producirse intoxicación por inyección subcutanea o intravenosa.
En algunos paises tales como Japón, Malasia, etc se trata de un producto frecuentemente utilizado con fines autolíticos $(2,3)$; pero en España existen muy pocos casos descritos de esta intoxicación.

\section{CASOS APORTADOS}

Presentamos dos casos de ingesta accidental de un herbicida. Los pacientes traian los restos del tóxico y por sus características se estableció la sospecha de que se trataba de paraquat. Los pacientes eran una mujer de 68 años de edad y su hijo de 50 años. Ambos habían ingerido el tóxico cuatro horas antes de su ingreso, aproximadamente unos 10-15 ml (un sorbo). A su llegada a urgencias presentaban sín-

Trabajo aceptado: 20 de diciembre de 2000

Correspondencia: Avda de la Mahía nº 106, edif. San José Portal 1, 3º-C. 15220. Bertamirans- Ames. La Coruña 
tomas de intenso dolor abdominal difuso, náuseas y vómitos, así como diarrea profusa, en la mujer estos síntomas eran más graves. Tras la recogida de muestras para toxicología se comienza con tratamiento específico, que posteriormente confirmaría la sospecha inicial. A su llegada el varón presentaba 11.000 leucocitos $(77 \% \mathrm{~N}$, 13L), Hb: 14,6, Hcto: 45,8, glucemia, urea, creatininina, bilirrubina, enzimas de colostasis y citolisis dentro de los límites normales. La mujer presentaba 8.000 leucocitos sin desviación izqda, función renal y hepática dentro de los límites normales. Tanto uno como el otro, a aire ambiente presentaban una saturación arterial de $99 \%$, por lo que no precisaron aporte de oxígeno. Se comienza inmediatamente con aporte intravenoso de $\mathrm{N}$-acetilcisteina como antioxidante $\mathrm{y}$ descontaminación intestinal con carbón activado y tierra de Fuller. Se añade además sulfato de magnesio como purgante y abundante hidratación con glucosa al $5 \%$ y se forzó diuresis con diuréticos de asa. Se comenzó rapidamente con la primera sesión de hemoperfusión con carbón activado, al término de ésta se retiraron nuevas muestras de plasma y orina. Se realizaron cuatro sesiones de tres horas de duración cada una, hasta que los niveles fueron practicamente indetectables en plasma. Los síntomas gastrointestinales comenzaron a ceder aproximadamente a las 6 horas del ingreso en el caso del varón, prolongándose unas 4 horas más en la mujer.

Presentamos la curva correspondiente a la evolución de los niveles en plasma y orina de ambos pacientes (Figs. 1 y 2 ).

Los pacientes permanecieron ingresados en la U.C.I durante 48 horas. Durante su ingreso no presentaron inestabilidad hemodinámica ni alteraciones en el gradiente a-A. En la Rx de tórax no se objetivó ninguna alteracion y su nivel de conciencia se mantuvo conservado en todo momento así como su función renal.

\section{DISCUSIÓN}

Se describen dos intoxicaciones por Paraquat; ambos pacientes bebieron de forma accidental aproximadamente entre 10 y $15 \mathrm{ml}$ del producto (un sorbo), que pudo llegar a ser fatal ya que dicho herbicida, utilizado para usos agrícolas se encuentra concentrado entre el 20 y el $30 \%$ (4).

Tras la ingesta el pico plasmático máximo se produce entre la hora y las dos horas y descienden rápidamente debido a su amplia distribución por los tejidos, el pulmón es el principal órgano diana ya que se produce acúmulo selectivo del tóxico, llegando a producir fibrosis pulmonar de rápida instauración, son afectados también resultan afectados los riñones, miocardio y tejido cerebral, pudiendo producirse edema. La vida media del Paraquat es de aproximadamente 12 horas, sin embargo en presencia de insuficiencia renal puede alargarse hasta 120 horas.

Los pacientes presentan en la mayoría de los casos sintomatología gastrointestinal, porque el Paraquat es extremadamente corrosivo, practicamente el $100 \%$ presentan vómitos, dolor abdominal y retroesternal. Si el paciente ha ingerido más de $40 \mathrm{mg} / \mathrm{kg}$ en la mayoría de los casos fallecerá entre las primeras horas y los cinco primeros días ya que desarrollaran fallo multiorgánico incluyendo SDRA, edema cerebral, necrosis miocárdica y fallo hepático.

Existen una serie de factores pronósticos, tales como el no desarrollo de ulceraciones gastricas o esofágicas en las primeras 24 horas se interpreta como un marcador de buen pronóstico; sin embargo la aparición de insuficiencia renal es por sí misma un indicador de mal pronóstico. Existen normogramas, el más utilizado es el de Hart que pone en relación la supervivencia con las concentraciones detectadas en plasma de este producto y con las horas transcurridas desde la ingesta del tóxico, es muy útil para diferenciar gru-

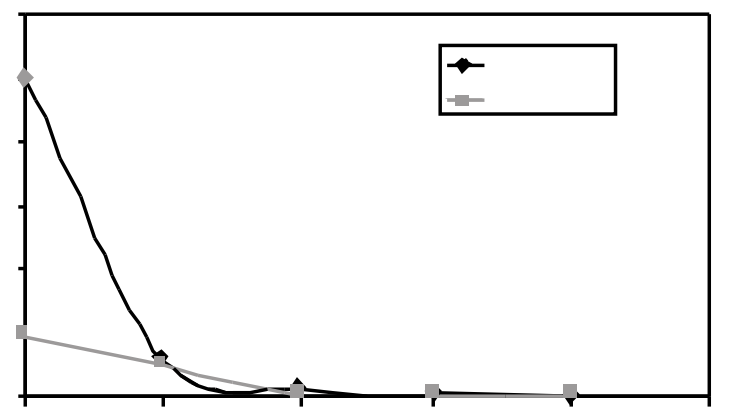

Fig. 1. Evolución de la concentración plasmática de Paraquat.



Fig. 2. Evolución de la concentración de Paraquat en orina.

pos pronósticos (5). En nuestros casos uno de ellos, el que presentaba síntomas más floridos presentaba una supervivencia aproximada de un $60 \%$.

Es muy importante, que la actitud a seguir ante la sospecha de una intoxicación por este producto se inicie de forma precoz y agresiva el tratamiento adecuado, comenzando con la descontaminación gastrointestinal con lavado gástrico con carbón activado y/o tierra de Fuller asociado a un catártico con el fin de ligar cualquier resto de Paraquat que exista en el tracto gastrointestinal; utilizar en combinación $\mathrm{N}$ acetil cisteina y desferoxamina como antioxidantes. No se ha demostrado que la diuresis forzada consiga de forma significativa un aumento en la eliminación del tóxico. Si han transcurrido menos de cinco horas de la ingestión se debe de comenzar con hemoperfusión con cartuchos de carbón activado, ya que ha demostrado ser más util que la hemodiálisis (6-8), al presentar mayor aclaramiento plasmático del tóxico. Debe de hacerse dentro de este rango horario porque es el tiempo en el que el paraquat alcanza su máxima concentración en plasma ya que posteriormente se distribuye en los tejidos. Existen algunos trabajos publicados en los que sin embargo no ha 
resultado util la hemodialisis sóla ni en combinación con hemoperfusión o técnicas dialíticas continuas (8). Algunos autores han abogado por la utilización de la plasmaféresis pero serían necesarios estudios más grandes para demostrar su beneficio.

Es importante intentar prevenir el daño pulmonar causado por este tóxico. El acumulo selectivo de paraquat en el tejido pulmonar es un proceso dependiente de energía. Los cambios producidos en el pulmón son muy similares al daño producido por la toxicidad debido a altas concentraciones de oxígeno. Se sabe que el aporte de oxígeno en este tipo de paciente acelera la patología pulmonar al producir radicales superoxido (9); es por tanto necesario mantener al paciente sin aporte de oxígeno y se aconseja sólo su uso por encima del $21 \%$ cuando la presión de oxígeno arterial dismiuya de $40 \mathrm{mmhg}$. Se publicó así mismo la utilización de oxido nítrico en este tipo de intoxicaciones, pero hasta el momento no ha sido util en los pocos pacientes en los que se ha utilizado.

Otras de las medidas terapéuticas que se han venido utilizando ha sido la administración de corticoesteroides con el fin de disminuir en la posible el daño pulmonar. Se han realizado estudios en ovejas en los que se ha podido observar que la administración de metilprednisolona tras la exposición al paraquat reduce la migración de prostaciclina al tejido pulmonara a traves del flujo linfático, sin embargo no reduce la acumulación de neutrófilos ni de tromboexano B2; en conejos la neumonitis por exposición al tóxico no fue prevenida ni su pronóstico fue mejorado con la administración de esteroides $(5,10)$. Se encuentra en fase experimental la posibilidad de utilización de inmunoterapia con fabricación de anticuerpos monoclonales específicos para la eliminación del paraquat del plasma.

Se podrían extraer como conclusiones que la ingestión de dosis mínimas de paraquat en un alto porcentaje de pacientes resultan mortales. Que se debe comenzar ante la sospecha de intoxicación por este producto con tratamiento específico de forma precoz y agresiva, ya que parece que la utilización de forma rápida (antes de las cinco horas post ingestión) de hemoperfución con carbón activado mejora la mortalidad de estos pacientes y que es importante la utilización de muy bajas concentraciones de oxígeno. Tanto los regimenes con inmunosupresores e inmunoterapia deben de realizarse estudios de más amplio número de pacientes para demostrar su utilidad.

\section{Bibliografía}

1. Camacho Pulido JA, Diaz Gomez A, Riquez Fernandez MD, Jurado Lara B, Jimenez Sanchez JM, Quesada Blanca JL. Poisoning by organophosphates and paraquat: a propos 14 cases. Rev Clin Esp 1995; 195 : 623-6.

2. Naito H, Yamashita M. Epydemiology of paraquat in Japan a new safe formulation of paraquat. Human toxicol 1987; 6: 87-8.

3. Bajo Bajo A, Sanz Ortega F, Santos Perez ME, Thonson Okatsu K, Zapico Alvarez N, García Perez A. Fatal Paraquat poisoning. An Med Interna (Madrid) 1996; 13: 79-80.

4. Yamashita M, Matsuo H. Analysis of 1.000 consecutive cases of acute poising in he suburb of Tokyo leading to hospitalization. Vet Hum Toxicol 1996; 38: 34-5.

5. William K, Chiang and rick Y, Wang. Pesticide poisoning. Little, Brown and company. James M. Rippe. $4^{\circ}$ th edition. New York. 1999, pp. 1765-7.

6. Lee CJ, Hsu HW, Chang YL. Performance characteristics of cambined haemodialysis/haemoperfusion system for removal $\mathrm{f}$ blood toxins. Med Eng Phys 1997; 19 (7): 658-67.

7. Drault JN, Baelen E, Medhdaoui H, Delord JM, Flament F. Massive paraquat poisoning. Favorable course after treatment with n-acetylcysteines and early hemodialysis. Ann Fr Anesth Reanim 1999; 18: 534-7.

8. Hampson EC, Pond SM. Failure of haemoperfusion and haemodialysis death in paraquat poisoning. Med Toxicol Adv Drug Exp 1988;3: 64.

9. Ayres JG, Lilford RJ. Treatment of paraquat poisoning. Thorax 1997; $52(6): 588$.

10. James F, Winchester MD. Paraquat and the bipyridyl herbicides. W.B. Saunders company. James Haddad. $3^{\circ}$ th edition. Philadelphia. 1990. 845-55.

11. Yang TS, Chang YL, Yen CK. Haemoperfusión treatment in pigs experimentally intoxicated by paraquat. Hum Exp Toxicol; 16 (12): 709-15. 